\title{
PROPOSTA DE CLASSIFICAÇÃO DA FAMÍLIA XANTHIDAE (CRUSTACEA, DECAPODA, BRACHYURA) ATRAVÉS DA TAXONOMIA NUMÉRICA
}

\author{
Petrônio Alves Coelho ${ }^{1}$ \\ Petrônio Alves Coelho Filho ${ }^{1}$
}

\begin{abstract}
PROPOSAL OF CLASSIFICATION OF THE FAMILY XANTHIDAE (CRUSTACEA, DECAPODA, BRACHYURA) BASED ON NUMERICAL TAXONOMY. Several families were added in the past in order to constitute the Xanthidae, but the boundaries between Xanthidae, Parthenopidae and Goneplacidae were diluted, and the classification of some genera doubtful. By this reason was made the analysis of a typical genus of each subfamily occurring in Brazil. 50 characters were selected and coded as $(0)$ for absence and (1) for presence. The data matrix was analysed by computer programs NTSYS and NMIX. The phenetic analysis shows the Xanthidae as a good family, but the cladistic one (Wagner tree) split them in four families: Carpiliidae, Xanthidae (with the subfamilies Menippinae, Platyxanthinae, Xanthinae and Eucratopsinae), Eriphiidae and Pilumnidae (with the subfamilies Trapeziinae and Pilumninae). Redescriptions and identifications keys for the proposed families and subfamilies are presented.
\end{abstract}

KEY WORDS. Brachyura, Xanthidae, systematic, numerical taxonomy

A classificação dos Decapoda em geral, e dos Brachyura em particular, é uma tarefa difícil, acerca da qual os especialistas dificilmente estão de acordo. A família Xanthidae, tal como é adimitida tradicionalmente (BALLS, 1957), é uma das mais numerosas entre os Brachyura, ultrapassando os Majidae e compreende 928 espécies no mundo (CHACE, 1951 apud GUINOT, 1978) e cerca de 100 espécies no Brasil, porém são muito difíceis de identificar, tanto para os carcinólogos, como para os biólogos em geral. Com efeito, como são numerosas e abundantes as espécies existentes em ambientes facilmente acessíveis, como os arrecifes e os estuários, ocorrendo desde a beira da praia até as maiores profundidades, seriam o objeto adequado para pesquisas fisiológicas, ecológicas, etc., porém as dificuldades na identificação são muito grandes, seja pela imprecisão das diagnoses dos gêneros e subfamílias, como pela falta de chaves atualizadas para as espécies.

Os limites entre as famílias Xanthidae, Parthenopidae e Goneplacidae nunca ficaram bem caracterizados, sendo discutida a posição sistemática de vários gêneros. Além disto, autoridades como ALCOOK (1893) e BORRA-

1) Departamento de Oceanografia, Universidade Federal de Pernambuco, Av. Prof. Morais Rego, 50670-420 Recife, Pernambuco, Brasil. Bolsista do CNPq. 
DAILE (1907) reuniram várias famílias para constituir os Xanthidae, situação que perdurou até 1978 quando GUINOT os elevou a superfamília, e os repartiu em oito famílias. Por outro lado, o trabalho de RATHBUN (1930) constitui a revisão mais recente para a família nas Américas, estando desatualizada quanto à constituição da fauna e distribuição das espécies.

Considerando todos estes fatos, foi sentida a necessidade da realização de uma revisão que redefinisse a família e as subfamílias, estabelecesse as relações de parentesco entre os diversos grupos, utilizando os métodos numéricos das análises fenética e filogenética.

Tabela I. Espécies representantes das subfamílias que acorrem no Brasil.

Família e subfamília

Espécie representante

GONEPLACIDAE

Casmocarcininae

Chasmocarcinus typicus Rathbun, 1898

Carcinoplacinae

Bathyplax typhla A. Milne Edwards, 1880

Geryoninae

Goneplacinae

Geryon ramosae Maninng; Tavares \& Albuquerque, 1989

Frevillea hirsuta (Borradaile, 1910)

Pseudorhombilinae

Pseudorhombila octodentata Rathbun, 1906

XANTHIDAE

Carpiliinae

Eriphiinae

Panopeinae

Platyxanthinae

Trapeziinae

Xanthinae

PARTHENOPIDAE

Aethrinae

Daldorfiinae

Parthenopinae
Carpilius corallinus (Herbst, 1783)

Eriphia gonagra (Fabricius, 1781)

Menippe nodifrons Stimpson, 1859

Panopeus lacustris Desbonne, 1867

Platyxanthus crenulatus A. Milne Edwards, 1879

Domecia acanthophora (Desbonne \& Schramm, 1867)

Cataleptodius floridanus (Gibbes, 1850)

Hepatus pudibundus (Herbst, 1785)

Thyrolambrus astroides Rathbun, 1894

Parthenope agona (Stimpson, 1871)

\section{MATERIAL E MÉTODOS}

O estudo foi iniciado pela revisão das amostras de Xanthidae, Parthenopidae e Goneplacidae reunidas ao longo dos anos no acervo da Coleção Carcinológica do Departamento de Oceanografia da Universidade Federal de Pernambuco e de parte da bibliografia pertinente sobre a ocorrência das espécies no Brasil e no mundo. Foi então verificado que grande parte das espécies conhecidas como ocorrendo no Brasil estavam presentes na referida coleção, porém havia várias lacunas, algumas delas potencialmente importantes do ponto de vista sistemático. Assim, foi realizado intercâmbio com o Museu de Zoologia da Universidade de São Paulo e a Coleção Carcinológica do Departamento de Oceaografia da Fundação Universidade Rio Grande, a fim de resolver este problema. 
Uma vez reunida coleção, o passo seguinte consistiu em rebaixar as famílias e superfamílias definidas por GUINOT (1978) e por NG \& RODRIGUEZ (1986) ao nível de subfamílias e famílias, respectivamente, voltando assim à condição em que são colocadas tradicionalmente. A seguir, foi realizada a análise morfológica de um representante típico de cada subfamília (Tab. I). Esta análise permitiu selecionar 50 caracteres morfológicos, relacionados na tabela II. A partir destes dados foi montada uma matriz, onde foi codificado "0" para a ausência e "1" para a presença de um caráter num táxon (Tab. III). Esta matriz serviu para analizar as relações fenéticas e filogenéticas das unidades taxonômicas, para o que foram empregados os programas dos pacotes NTSYSPC e NMIX.

$\mathrm{Na}$ análise fenética foi adotado o coeficiente de semelhança simples, ponto de partida para agrupar as OTUs pelos métodos da ligação simples, da ligação completa e da ligação média não ponderada. $\mathrm{Na}$ análise cladística foi usada a opção GLOBAL, porém acrescentando uma OTU hipotética considerada como ancestral.

O pacote NTSYS-PC (Numerical Taxonomy System) é uma versão "micro" do NTSYS, escrita para ser usada em microcomputadores IBM PC, XT, AT, PS/2 e compatíveis, enquanto o pacote NMIX (MIX Method of Parsimony) utiliza os métodos de CAMIM-SOKAL e de WAGNER para reconstrução de filogenias.

Foi escolhida uma das árvores de Wagner obtidas, definindo então as familias e subfamílias propostas no item resultados. Foram também redigidas chaves para identificação das famílias e subfamílias e efetuada uma tentativa de classificação dos gêneros da fauna brasileira.

\section{RESULTADOS}

Análise fenética. As figuras 1,2 e 3 mostram os dendrogramas resultantes desta análise. Nos dendrogramas originados é possível reconhecer claramente os Xanthidae como um agrupamento válido. Nas três classificacões Eriphia Latreille, 1817 está distante de Menippe De Haan, 1893, sendo necessário separá-los como Eriphiinae e Menippinae; os Geryonidae estão excluídos dos Xanthidae, porém, no seio da família, os Trapeziinae e Pilumnidae, de um lado, e os Xanthinae + Eucratopsinae + Platyxanthinae + Menippinae, de outro, constituem grupos constantes, enquanto os Eriphiinae em duas classificações estão unidos aos Trapeziinae e Pilumninae, e na terceira aos Xanthinae + Eucratopsinae + Platyxanthinae + Menippinae.

Análise cladística. As figuras 4 e 5 mostram as duas árvores de Wagner, que são bastante semelhantes entre si. Em ambas, os Xanthidae da classificação fenética desaparecem como agrupamento monofilético, formando assim quatro famílias.

De acordo com o presente estudo é proposta a seguinte classificação para o grupo estudado: Carpiliidae, não dividida em subfamílias; Xanthidae (latu sensu), com as subfamílias Menippinae, Platyxanthinae, Xanthinae e Eucratop- 
sinae; Eriphiidae, não dividida em subfamílias e Pilumnidae, com Trapeziinae e Pilumninae (Fig. 6).

Tabela II. Lista dos caracteres selecionados para estudo.

\begin{tabular}{|c|c|}
\hline Caracteres & Caráter no estado "1" (presença) \\
\hline 01 & Regiões da carapaça bem marcadas \\
\hline 02 & Formato da carapaça oval ou transversalmente oval \\
\hline 03 & Carapaça lisa (sem espinhos ou grânulos) \\
\hline 04 & Superfície da carapaça com pêlos \\
\hline 05 & Carapaça dividida em dois bordos \\
\hline 06 & Margem posterior da carapaça com duas expansões \\
\hline 07 & Carapaça e quelípedes profundamente sulcados e erodidos \\
\hline 08 & Margem ântero-lateral com espinhos ou dentes \\
\hline 09 & Margem ântero-lateral bastante arqueada \\
\hline 10 & Margem ântero-lateral maior que as póstero-laterais \\
\hline 11 & Margem ântero-lateral com dois espinhos além do orbital \\
\hline 12 & Margem ântero-lateral com quatro espinhos além do orbital \\
\hline 13 & Margem ântero-lateral com quatro projeções, somente as duas últimas dentiformes \\
\hline 14 & Margem ântero-lateral com apenas um tubérculo lateral \\
\hline 15 & Margem ântero-lateral dirigindo-se para a cavidade bucal \\
\hline 16 & Bordo fronto-orbital mais da metade da maior largura da carapaça \\
\hline 17 & Fronte com rostro \\
\hline 18 & Rostro dobrado para baixo, não visto dorsalmente \\
\hline 19 & Órbitas alongadas \\
\hline 20 & Margens orbitais com entalhes \\
\hline 21 & Margens orbitais com espinhos \\
\hline 22 & Antenas excluídas das órbitas \\
\hline 23 & Pedúnculos oculares com estruturas \\
\hline 24 & Antênulas dobrando transversalmente \\
\hline 25 & Configuração da cavidade bucal semi-quadrada \\
\hline 26 & Maxilípedes externos cobrindo todo o espaço da cavidade bucal \\
\hline 27 & Maxilípedes externos lisos \\
\hline 28 & Comprimento do mero dos maxilípedes externos maior que a largura \\
\hline 29 & Comprimento do mero dos maxilípedes externos igual à largura \\
\hline 30 & Carpo, propódio e dáctilo dos maxilípedes externos sob o mero \\
\hline 31 & Quelípedes grandes, menos que duas vezes o comprimento da carapaça \\
\hline 32 & Quelípedes e/ou dedos fortes \\
\hline 33 & Quelípedes lisos, sem estruturas \\
\hline 34 & Quelípedes e patas ambulatórias com pêlos \\
\hline 35 & Quelípede esquerdo totalmente diferente do direito \\
\hline 36 & Dedos da quela escuros \\
\hline
\end{tabular}

Cont. 
Tabela II. Continuação

\begin{tabular}{cl}
\hline Caracteres & \multicolumn{1}{c}{ Caráter no estado "1" (presença) } \\
\hline 37 & Coloração dos dedos continuando para as palmas \\
38 & Dedos da quela longos \\
39 & Extremidade distal dos dedos da quela agudas \\
40 & Dedos encurvados para baixo \\
41 & Patas ambulatórias fortes e curtas \\
42 & Patas ambulatórias lisas, sem estruturas \\
43 & Segmentos do abdômen fusionados \\
44 & Terceiro segmento do abdômen do macho tocando o quinto par de patas \\
45 & Télson quase tocando o terceiro esternito torácico \\
46 & Tórax alargado \\
47 & Suturas entre os esternitos do macho incompletas \\
48 & Oitavo esternito do macho visível \\
49 & Primeiro par de pleópodos do macho mais longo que o segundo par \\
50 & Segundo par de pleópodos do macho mais longo que o primeiro par \\
\hline
\end{tabular}

Tabela III. Matriz de dados das espécies representantes das subfamílias estudadas.

\begin{tabular}{llllll}
\hline \multicolumn{1}{c}{ OTUs } & & & Caracteres & & \\
\hline Ancestral & 0110100111 & 0000000000 & 0011110001 & 1100100101 & 101000000 \\
Pseudorhombila & 1000100100 & 0100010001 & 0001100101 & 0000001111 & 010111100 \\
Geryon & 1000100100 & 0000010001 & 1001110001 & 1000000110 & 000111100 \\
Chasmocarcinus & 0010000000 & 0000010000 & 0001011001 & 0100001110 & 110111100 \\
Bathyplax & 0000100100 & 1000010000 & 0011010001 & 0001011110 & 000111110 \\
Menippe & 1110100111 & 0000000000 & 0001110001 & 1100100101 & 101001010 \\
Platyxanthus & 1110100111 & 0010000000 & 0001110000 & 1100100101 & 101001010 \\
Panopeus & 1110100100 & 0000010000 & 0011110000 & 1100100101 & 111001010 \\
Cataleptodius & 1110100111 & 0000010000 & 0001110100 & 1100110001 & 111001010 \\
Carpilius & 0110100011 & 0001000000 & 0001110101 & 1100100101 & 111000000 \\
Eriphia & 1000100100 & 0000010000 & 1011100001 & 1000100101 & 101001000 \\
Domecia & 0001100100 & 0000010001 & 1001100100 & 1000110101 & 001001010 \\
Pilumnus & 0101100100 & 0000010001 & 0001110100 & 1010100101 & 001001000 \\
Hepatus & 0110110111 & 0000100000 & 0000101010 & 1000000101 & 111101001 \\
Thyrolambrus & 1000111101 & 0000001100 & 0101100110 & 0000000101 & 001101000 \\
Parthenope & 1000110101 & 0000001001 & 0101100100 & 0000000100 & 111101000 \\
Fervilea & 0010000000 & 0000010010 & 0011110101 & 0000001110 & 101111010 \\
\hline
\end{tabular}



0.400
0.500
0.600
0.700
0.800
0.900
1.000

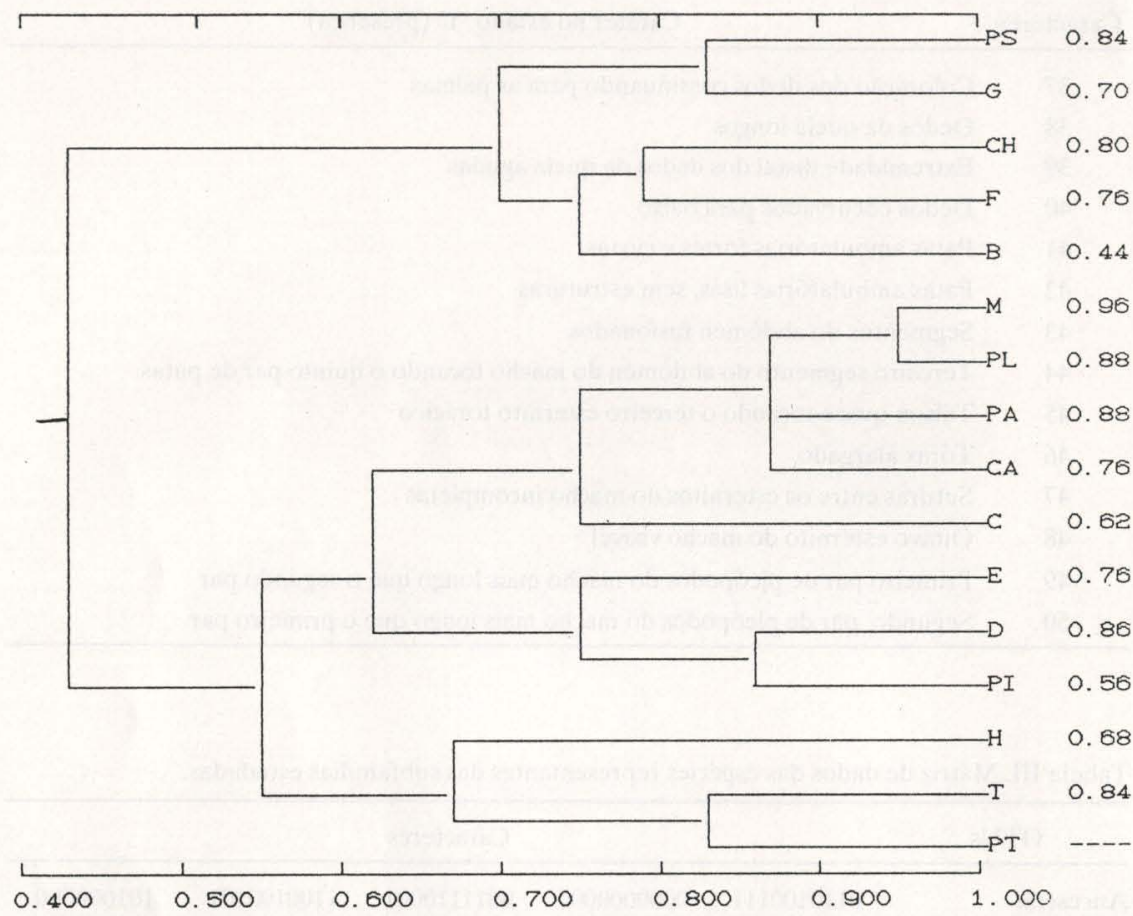

Fig. 1. Dendrogramas resultantes da análise fenética, através do Agrupamento das subfamílias pelo método da ligação completa. (PS) Pseudorhombilinae; (G) Geryoninae; (B) Carcinoplacinae; (CH) Chasmocarcininae; (F) Euryplacinae; (M) Menippinae; (PL) Platyxanthinae; (PA) Eucratopsinae; (CA) Xanthinae; (C) Carpiliinae; (E) Eriphiinae; (D) Trapeziinae; (PI) Pilumninae; (H) Aethrinae; (T) Daldorfiinae; (PT) Parthenopinae.

\section{Chave para a identificação das famílias estudadas}

1. Carapaça, quelípedes e patas ambulatórias com pêlos e espinhos, ou pelo menos carapaça recoberta por pêlos ................ Pilumnidae

- Carapaça, quelípedes e patas ambulatórias sem pêlos e espinhos ........2

2. Margem posterior da carapaça com duas expansões ..........Parthenopidae

- Margem posterior da carapaça desprovida de expansões . . . . . . . . . . 3

3. Carapaça subquadrada ou trapezoidal .................... 4

- Carapaça oval ou transversalmente oval ..................... 5

4. Carapaça com sete dentes ântero-laterais; quelípedes fortes, com a face externa coberta por grânulos grandes e arredondados; dedos das quelas escuros; oitavos esternitos ocultos; esterno alongado........ Eriphiidae 
- Carapaça com até cinco dentes ântero-laterais; quelípedes geralmente finos e encurvados para baixo, sem grânulos arredondados; dedos das quelas brancos; oitavo esternito visível; quando não, segundo segmento do abdômen de largura semelhante ao terceiro; esterno arredondado e alargado ............................... Goneplacidae

5. Carapaça transversalmente oval, sem traços de regiões; margens ânterolaterais não recortadas, com apenas um tubérculo no ângulo lateral; antenas excluídas das órbitas .................... Carpiliidae

- Carapaça hexagonal; regiões bem marcadas; margens ântero-laterais recortadas em dentes, espinhos ou tubérculos; antenas incluídas nas órbitas . Xanthidae

\section{Carpiliidae Ortmann, 1893}

Carpiliinae Ortmann, 1893:409. - Guinot, 1968:320.

Carpiliidae; Guinot, 1978:267.

Redescrição. Carapaça oval, lisa, sem espinhos, grânulos ou pêlos; regiões mal definidas. Margem posterior desprovida de espansões; margens ântero-laterais bastante arqueadas, desprovidas de espinhos ou dentes, apenas com um tubérculo lateral; bordo fronto orbital menos da metade da maior largura da carapaça. Órbitas com margens desprovidas de entalhes. Antenas excluídas das órbitas. Maxilípedes externos lisos; mero mais largo do que longo. Quelípedes grandes, fortes, lisos, porém menos de duas vezes o comprimento da carapaça; dedos escuros, não encurvados. Patas ambulatórias lisas e cilíndricas. Esterno estreito em relação à carapaça, sua cavidade não continuando em direção à cavidade bucal; suturas entre os esternitos paralelas e completas; oitavo esternito do macho oculto. Primeiros segmentos do abdômen fusionados. Télson distante da margem posterior do terceiro esternito. Pleópodos do primeiro par, nos machos, de comprimento semelhante aos do segundo par.

Subfamílias incluídas: não dividida em subfamílias.

Gênero incluído: Carpilius Leach, 1825 (Fig. 8d).

\section{Xanthidae Mac Leay, 1838}

Xanthidae Mac Leay, 1838:59.

Redescrição. Carapaça oval ou transversalmente oval, desprovida de espinhos, grânulos e pêlos; regiões bem marcadas; margem posterior desprovida de expansões; margens ântero-laterais com espinhos ou dentes. Antenas não excluídas das órbitas. Maxilípedes externos lisos. Quelípedes grandes, com estruturas, porém menos de duas vezes o comprimento da carapaça; dedos escuros, não encurvados para baixo; cavidade esternal não continuando em direção à cavidade bucal. Suturas entre os esternitos do macho incompletas e convergentes; esterno estreito em relação à carapaça; oitavo esternito do macho 
oculto. Pleópodos do primeiro par, no macho, mais longos que os do segundo par.

Subfamílias incluídas: Platyxanthinae, Menippinae, Eucratopsinae e Xanthinae.
0. 700
0. 750
0.800
0.850
0.900
0.950
1.000

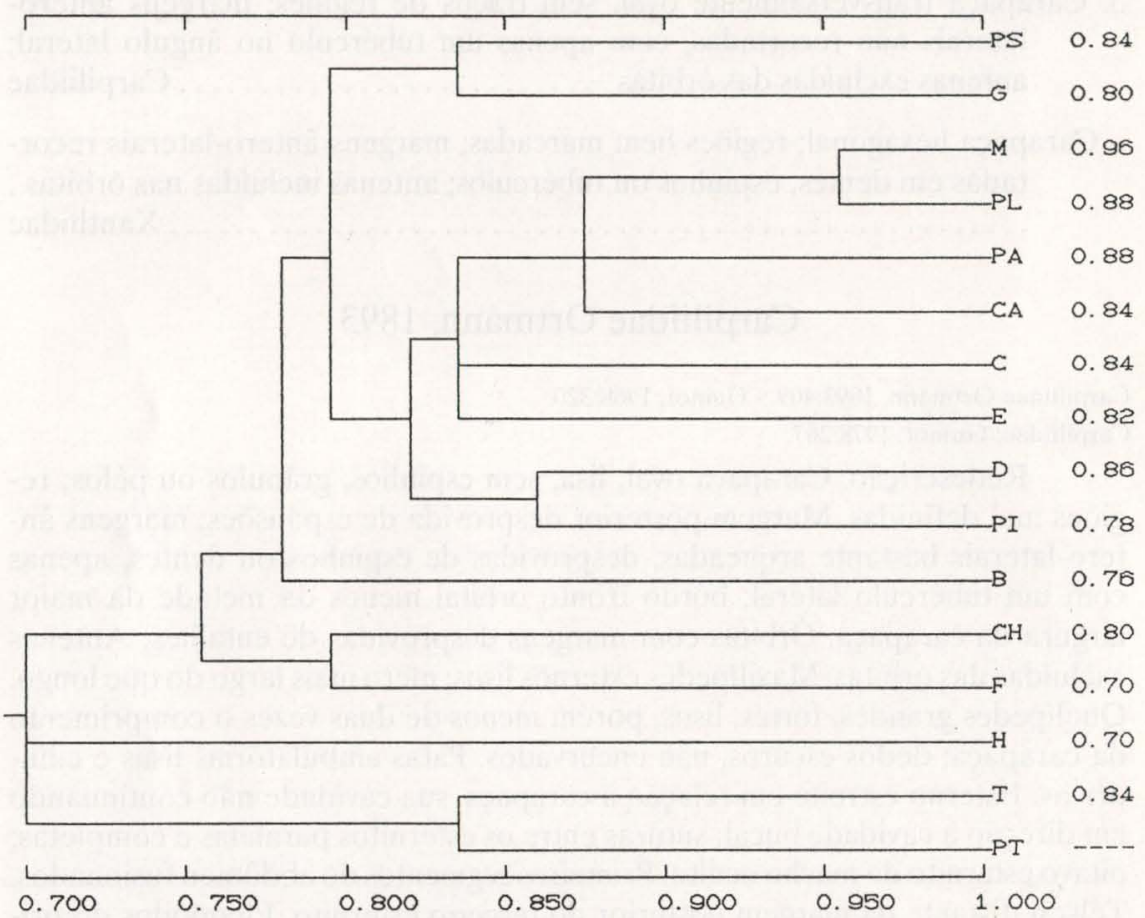

Fig. 2. Dendrogramas resultantes da análise fenética. Agrupamentos das subfamilias pelo método da ligação simples; coeficiente de semelhança simples. Símbolos como na figura 1.

\section{Chave para identificação das subfamílias}

1. Bordo fronto-orbital menos da metade da maior largura da carapaça; todos os segmentos do abdômen do macho livres................. 2

- Bordo fronto-orbital mais da metade da maior largura da carapaça; alguns segmentos do abdômen do macho fusionados ................ 3

2. Projeções ântero-laterais não distintamente dentiformes, podendo ser fusionadas, subdivididas ou recortadas; fronte quadridentada; suturas entre os esternitos do macho bastante confluentes .......... Platyxanthinae

- Cinco projeções ântero-laterais dentiformes; fronte bidentada; suturas entre os esternitos do macho pouco confluentes ............. Menippinae 
3. Margens ântero-laterais bastante arqueadas, maiores que os póstero-laterais; antênulas dobradas obliquamente; extremidade dos dedos da quela maior e/ou menor em forma de colher, quando não, quelípedes com espinhos ou tubérculos ......................... Xanthinae

- Margens ântero-laterais levemente encurvadas, com tamanho semelhante ou menor que as póstero-laterais; antênulas dobradas transversalmente; extremidade distal dos dedos aguda ............... Eucratopsinae
0.560
0. 640
0.720
0.800
0.880
0.960
1.040

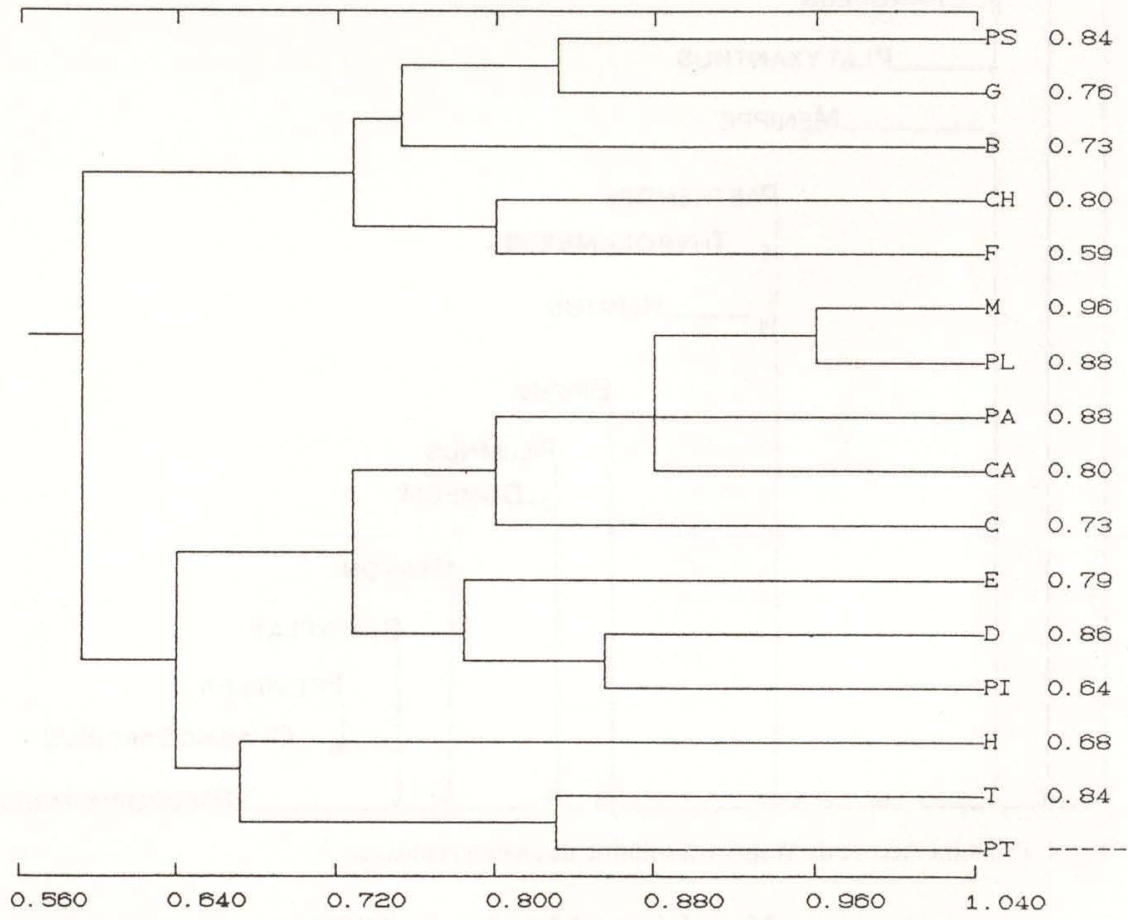

Fig. 3. Dendrogramas resultantes da análise fenética. Agrupamento das subfamilias pelo método da ligação média não ponderada. Símbolos como na figura 1.

\section{Platyxanthinae Guinot, 1977}

Platyxanthinae Guinot, 1977:1052.

Redescrição. Margens ântero-laterais da carapaça bastante arqueadas, maiores que as póstero-laterais, armadas com projeções não distintamente dentiforme, podendo ser reduzidos, fusionados, subdivididos ou ausentes. Margens orbitais com entalhes; bordo fronto-orbital menos da metade da maior largura da carapaça; fronte quadridentada. Antênulas dobradas obliquamente. Maxilípedes externos com mero de comprimento semelhante à largura. Abdô- 
men com todos os segmentos livres.

Gêneros incluídos: Homalaspsis A. Milne Edwards, 1863; Pelaeus Eudoux \& Souleyet, 1842; Platyxanthus A. Milne Edwards, 1863 (Fig. 7d,e).

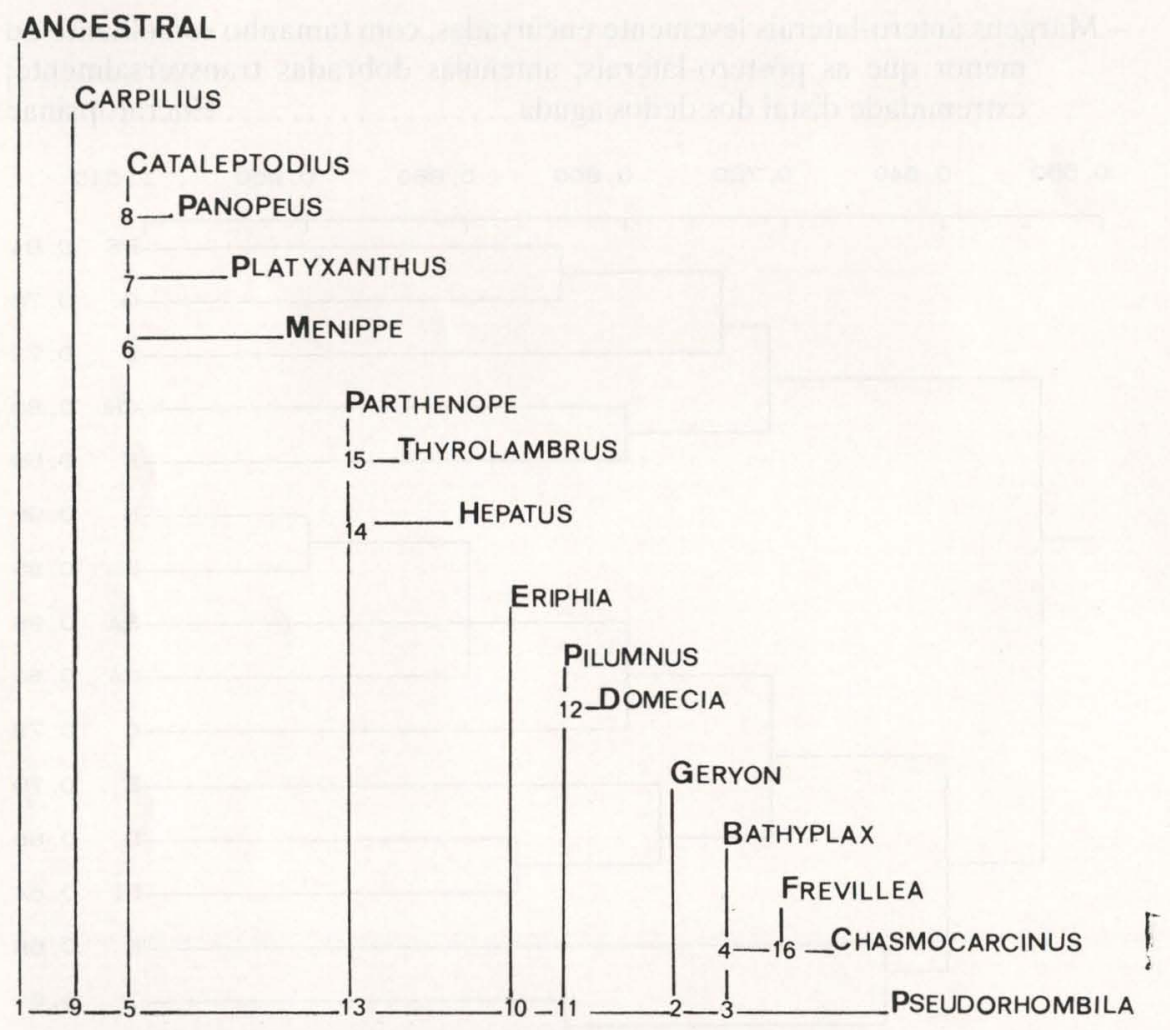

Fig. 4. Primeira Arvore de Wagner resultante da análise cladistica.

\section{Xanthinae Mac Leay, 1838}

Xanthinae Mac Leay, 1838:59.

Redescrição. Margens ântero-laterais bastante arqueadas, maiores que as póstero-laterais; bordo fronto-orbital mais da metade da maior largura da carapaça; margens orbitais com entalhes. Antênulas dobradas obliquamente. Extremidade distal dos dedos de pelo menos uma das quelas em forma de colher; se não, quelípedes possuindo espinhos ou tubérculos. Maxilípedes externos com o comprimento do mero nitidamente inferior à largura. Alguns segmentos do abdômen fusionados.

Gêneros incluídos: Actaea De Haan, 1833; Allactaea Williams, 1974; Banareia A. Milne Edwards, 1869; Cataleptodius Guinot, 1967 (Fig. 7c); Edwardsium Guinot, 1967; Euryozius Miers, 1886; Paractaea Guinot, 1969; 
Platypodiella Guinot, 1967; Pseudomedaeus Guinot, 1968; Tetraxanthus Rathbun, 1898; Xanthias Rathbun, 1897; Xanthodius Stimpson, 1859.

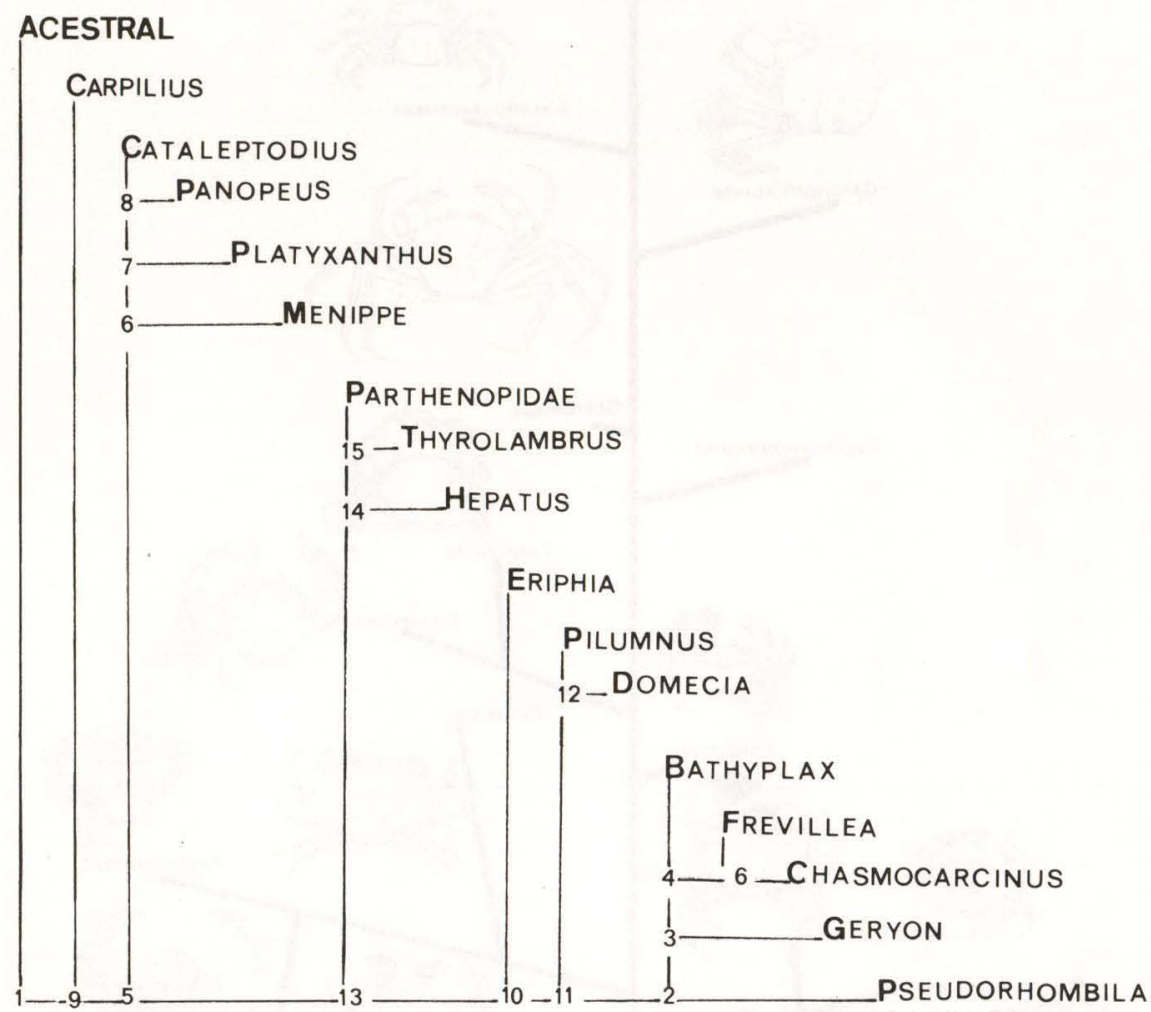

Fig. 5. Segunda Árvore de Wagner obtida na análise cladística. Esta foi a árvore ecolhida para definição das famílias e subfamílias.

\section{Menippinae Ortmann, 1893}

Menippinae Ortmann, 1893:428.

Redescrição. Margens ântero-laterais da carapaça bastante arqueadas, maiores que as póstero-laterais; bordo fronto-orbital menos da metade da maior largura da carapaça; margens orbitais sem entalhes. Antênulas dobradas obliquamente. Maxilípedes externos com mero de comprimento semelhante à largura. Quelípedes grandes, menos de duas vezes o comprimento da carapaça; extremidades dos dedos da quela aguçadas. Abdômen com todos os segmentos livres.

Gênero incluído: Menippe De Haan, 1893 (Fig. 7b). 


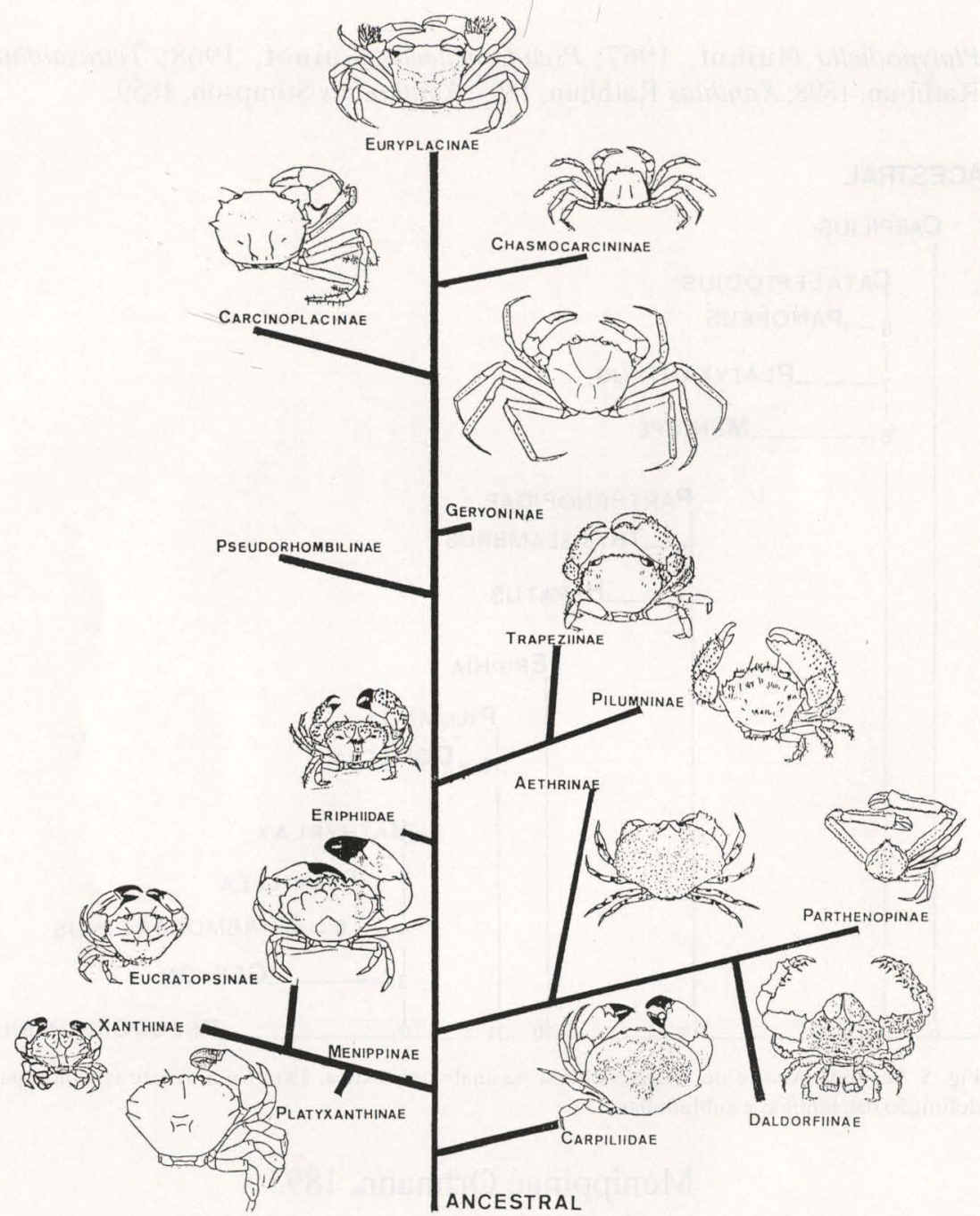

Fig. 6. Representação esquemática da filogenia do grupo estudado.

\section{Eucratopsinae Stimpson, 1871.}

Eucratopsinae Stimpson, 1871:151.

Redescrição. Margens ântero-laterais pouco arqueadas, iguais ou menores que as póstero-laterais; bordo fronto-orbital mais da metade da maior largura da carapaça; margens orbitais com entalhes. Antênulas dobradas transversalmente. Maxilípedes externos com mero de comprimento semelhante à largura. Quelípedes relativamente pequenos; extremidade distal dos dedos das quelas agudas. Alguns segmentos do abdômen do macho fusionados. 

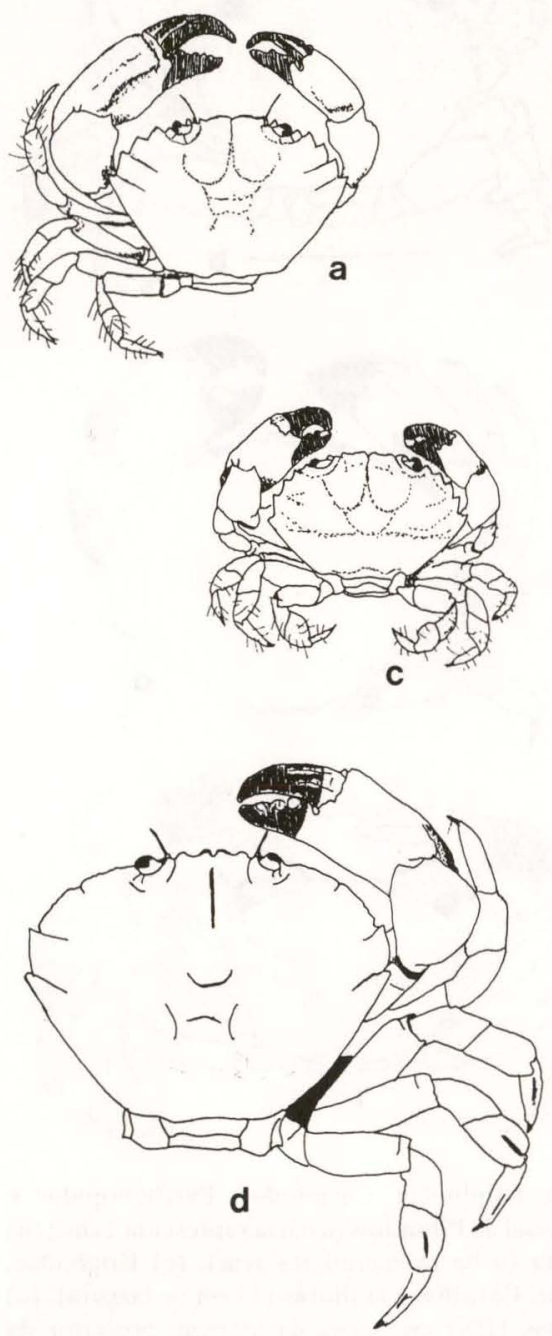

Fig. 7. Representantes típicos de Xanthidae. (a) Eucratopsinae, vista dorsal de Panopeus ( $5 \mathrm{~cm}$ de largura); (b) Menippinae, vista dorsal de Menippe nodifrons (a barra representa $1 \mathrm{~cm}$ ); (c) Xanthinae, vista dorsal de Cataleptodius floridanus (3,5 cm de largura); (d) Platyxanthinae, Platyxanthus crenulatus; (e) esterno de Platyxanthus crenulatus; números dos esternitos em arábicos; (A1) Primeiro segmento abdominal; (OM) orifício genital masculino. (a, c) Modificado de CHACE et al. (1986); (b) modificado de ABELE \& KIN, 1986; (d) original de COELHO FILHO; (e) modificado de GUINOT, 1976. 

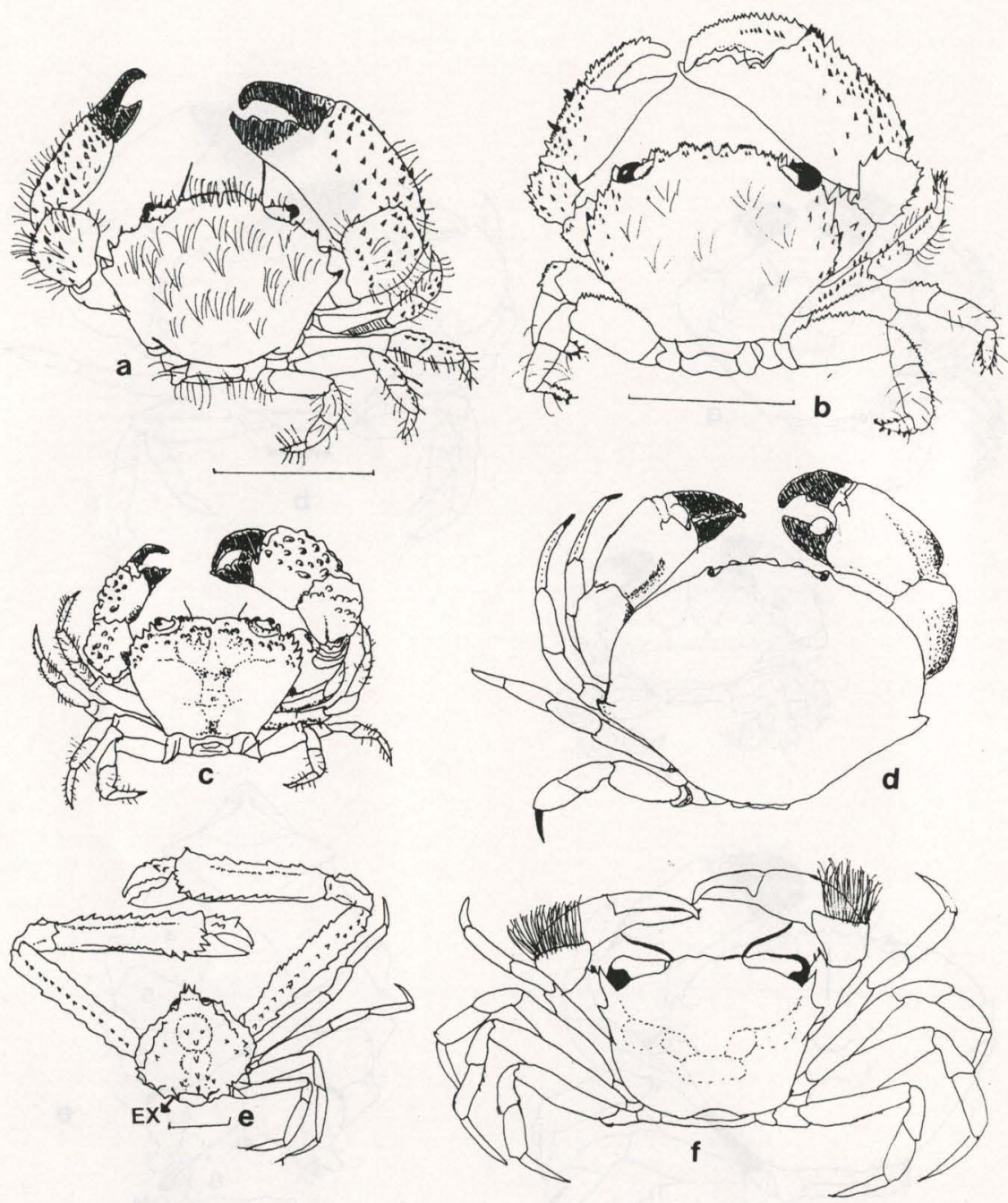

Fig. 8. Representantes típicos de Pilumnidae, Eriphiidae, Carpiliidae, Parthenopidac e Goneplacidae. (a) Pilumnidae, Pilumninae, vista dorsal de Pilumnus (a barra representa $1 \mathrm{~cm}$ ); (b) Pilumnidae, Trapeziinae, Domecia acanthophora (a barra representa $1 \mathrm{~cm}$ ); (c) Eriphiidae, Eriphia gonagra (4 cm de largura); (d) Carpiliidae, Carpilius corallinus (15 cm de largura); (e) Parthenopidae, Parthenopinae, Parthenope agona, (EX) expansões da margem posterior da carapaça. (f) Goneplacidae, Goneplacinae, Frevilea hirsuta (2 $\mathrm{cm}$ de largura). (a, b, e, f) Modificados de ABELE \& KIN (1986); (c, d) modoficados de CHACE et al. (1986).

Gêneros incluídos: Cyrtoplax Rathbun, 1914; Eucratopsis Smith, 1869; Eurypanopeus A. Milne Edwards, 1880; Eurytium Stimpson, 1859; Hexapanopeus Rathbun, 1898; Panopeus Milne Edwards, 1834 (Fig. 7a); Rhitropanopeus Rathbun, 1898. 


\section{Pilumnidae Samouelle, 1819}

Pilumnidae Samouelle, 1819:86.

Redescrição. Regiões da carapaça mal delimitadas; carapaça e patas ambulatórias com pêlos, além de espinhos ou grânulos; margem posterior da carapaça desprovida de expansões; margens ântero-laterais com espinhos ou dentes, pouco arqueadas, menores que as póstero-laterais; bordo fronto-orbital mais da metade da maior largura da carapaça; margens orbitais com entalhes. Maxilípedes externos com mero de comprimento semelhante à largura. Quelípedes pequenos, ásperos, com estruturas; dedos das quelas escuros, não encurvados para baixo. Suturas entre os esternitos confluentes, incompletas. Cavidade onde se aloja o abdômen não continuando em direção à cavidade bucal; abdômen com todos os segmentos livres; télson distante do terceiro esternito torácico. Esterno estreito em relação à carapaça; oitavos esternitos do macho ocultos.

Subfamílias incluídas: Trapeziinae e Pilumninae.

\section{Chave para identificação das subfamílias}

1. Carapaça subquadrada; antenas excluídas das órbitas; maxilípedes externos não cobrindo todo o espaço bucal e com espinhos; quelípedes e patas ambulatórias sem pêlos ........................ Trapeziinae

- Carapaça transversalmente oval; antenas incluídas nas órbitas; maxilípedes externos lisos e cobrindo todo o espaço da cavidade bucal; quelípedes e patas ambulatórias peludos ..................... Pilumninae

\section{Pilumninae Samouelle, 1819}

Pilumnidae Samouelle, 1819:86.

Redescrição. Carapaça de formato transversalmente oval; antenas não excluídas das órbitas; maxilípedes externos lisos; extremidades dos dedos das quelas agudos; patas ambulatórias e quelípedes com espinhos e pêlos; pleópodos do segundo par do macho de comprimento semelhante aos do primeiro.

Gênero incluído: Pilumnus Leach, 1815 (Fig. 8a).

\section{Trapeziinae Miers, 1886}

Trapeziinae Miers, 1886:163.

Redescrição. Carapaça de formato mais ou menos trapezoidal; antenas excluídas das órbitas; maxilípedes externos com espinhos; extremidades dos dedos das quelas em forma de colher; patas ambulatórias lisas; primeiro par de pleópodos do macho mais longos que os do segundo par.

Gêneros incluídos: Domecia Eydoux \& Souleyt, 1842 (Fig. 8b); Micropanope Stimpson, 1871:139. 


\section{Eriphiidae Mac Leay, 1838}

Eriphiidae Mac Leay, 1838:59. - Dana, 1852:145.

Redescrição. Carapaça não oval, áspera, com espínulos ou grânulos; com regiões bem definidas; margens ântero-laterais com espinhos ou dentes, pouco arqueadas, iguais ou menores que as póstero-laterais; bordo fronto-orbital mais da metade da maior largura da carapaça. Órbitas com entalhes. Antenas excluídas das órbitas. Maxilípedes externos ásperos, comprimento do mero semelhante à sua largura. Quelípedes fortes e ásperos, menos de duas vezes o comprimento da carapaça; dedos das quelas escuros, não recurvados para baixo. Patas ambulatórias lisas. Todos os segmentos do abdômen livres. Télson distante do terceiro esternito; suturas do esterno incompletas, convergentes; oitavo esternito do macho oculto; esterno estreito em relação à carapaça. Pleópodos do macho de tamanho semehante.

Gênero incluído: Eriphia Latreille, 1817 (Fig. 8c).

\section{DISCUSSÃO}

De acordo com os feneticistas, as classificações devem estar apoiadas na análise da similarididade, porém o número de taxa é arbitrário, desde que esteja coerente com os resultados obtidos. Por outro lado, para os seguidores do cladismo, a classificação deve refletir a seqüência de ramificações do cladograma. Desta forma, um primeiro critério para avaliação da classificação obtida é o da coerência entre os resultados das análises fenética e cladística. Um outro critério é o da comparação com as classificações (quase sempre evolucionistas) estabelecidas por outros pesquisadores.

Os agrupamentos observados na análise fenética se repetiram geralmente na análise cladística, como é possível observar comparando-se os dendogramas. Isto pode ser aceito como argumento a favor da validez da classificação obtida. É possível prever também que, sendo empregados os mesmos caracteres, os demais Cancroidea encontrariam seu lugar nos dois esquemas.

Os Cancroidea foram definidos por DANA (1852) levando em consideração uma série de caracteres da região frontal, brânquias, carapaça, antênulas, antenas e aberturas genitais. Na presente análise, os caracteres apomorfos que uniram todos os grupos estudados foram ligados às antênulas e patas ambulatórias. Os demais caracteres assinalados por DANA (op. cit.) são considerados plesiomorfismos, não os discriminando de grupos situados anteriormente na árvore filogenética. Apesar disto, não seria fora de propósito a procura de outras apomorfias para o grupo.

O ponto $9 \mathrm{da}$ árvore serve de argumento para indicar que os Xanthoidea, tal como definidos por GUINOT (1976), constituem um agrupamento que poderia ser mantido feneticamente, porém que não se justificariam filogeneticamente, apesar do desejo da autora de propor um agrupamento monofilético. Desta forma, os Carpiliidae se dissociaram por quatro apomorfias, enquanto duas outras definiram os demais caranguejos estudados. 
O ponto 6 indica que os Menippinae, Platyxanthinae, Eucratopsinae e Xanthinae constituem um agrupamento monofilético, para o qual, de acordo com as Regras de Nomenclatura Zoológica em vigor, existe disponível o nome de Xanthidae. O caráter que os reúne está ligado aos pleópodos do macho; neste caso, é urgente a necessidade de procurar novas sinapomorfias, capazes de melhor definir o grupo, com relação aos seus parentes mais próximos.

O ponto 10 separa Eriphia gonagra (Fabricius, 1781) dos demais, pelo carácter relacionado à inclusão do pedúnculo antenal no hiato orbital.

$\mathrm{O}$ ancestral 11 originou duas linhagens bem características, uma dos Pilumnidae, pelo relevo da carapaca, pilosidade, maxilípedes externos e quelípedes; e a dos Goneplacidae, pela coloração e formato dos dedos, terceiro segmento do abdômen, télson e esternos torácicos.

Ao contrário, os Parthenopidae, cujo ancestral está descrito no ponto 14, com pelo menos quatro sinapomorfias, parece um grupo muito bem caracterizado. A posição sistemática dos Aethrinae que, como GUINOT acentıou em vários trabalhos publicados entre 1966 e 1978, estava dispersa entre os Parthenopidae, Calappidae e Leucosiidae, agora recebe mais uma confirmação da sua classificação entre os Parthenopidae.

Um argumento em apoio à classificação obtida é encontrado no estudo das larvas. RICE (1980), reconheceu quatro grupos de larvas de Xanthidae, os quais não coincidiam com as divisões de GUINOT (1978). Esta classificação foi aperfeiçoada por MARTIN (1984), elevando o número de grupos para oito. Entre os gêneros aqui estudados, Panopeus Milne Edwards, 1834 e Cataleptodius Guinot, 1967, apresentam larvas classificadas no Grupo I; Pilumnus no Grupo II; Eriphia Latreille, 1817 e Platyxanthus A. Milne Edawrds, 1863, no Grupo III, e Menippe De Hann, 1893, no Grupo IV. O Grupo V não interessa no momento e o Grupo VI encerra apenas Panopeus bermudensis Benedict \& Rathbun, 1891, como exceção ao Grupo I. FRANSOZO (1987) confirmou que as larvas de Eriphia e Menippe pertencem a grupos diferentes. Desta forma, em suas linhas gerais, a divisão dos Xanthidae em quatro famílias poderia encontrar confirmação em dados obtidos no estudo do desenvolvimento larval.

A maioria dos gêneros pertencentes aos Xanthidae de GUINOT (vários trabalhos) estão bem caracterizados na subfamília Xanthinae, como está sendo presentemente adotada, ficando pequenas dúvidas em relação a alguns gêneros como Euryozius Miers, 1886, Tetraxanthus Rathbun, 1898, Pseudomedaeus Guinot, 1968 e afins, e Edwardsium Guinot, 1967.

A família Menippidae continha, entre outros, os gêneros Menippe De Haan, 1893 e Eriphia Latreille, 1817. A partir da análise cladística efetuada, estes dois gêneros localizaram-se em monofilias diferentes. Menippe ficou caracterizado entre os Xanthidae, compondo a subfamília Menippinae e Eriphia ficou isolada em uma monofilia, sendo para este utilizada a família Eriphiidae.

Ao formar a família Panopeidae, GUINOT (1978) incluiu a maior parte dos gêneros classificados antes na subfamília Prionoplacinae (Goneplacidae). Estes gêneros, que constituem a linhagem goneplacidiana dentro dos panopeí- 
deos, formam um conjunto que nunca foi bem caracterizado, sendo questionado por vários autores, como MARTIN \& ABELE (1986), que analisando a forma dos pleópodos do macho e comparando-as com as de Panopeus, assinalaram algumas diferenças. Na presente análise, este grupo fica caracterizado como uma subfamília de Xanthidae, porém nem todos os gêneros prionoplacíneos estão bem classificados como Xanthidae. No presente momento estão sendo aceitos no grupo apenas os gêneros: Rhithropanopeus Rathbun, 1898, cujos pleópodos são diferentes dos de Panopeus Milne Edwards, 1834, mas externamente são muito parecidos; Cyrtoplax Rathbun, 1914 que é bastante semelhante aos Goneplacidae, porém seus pleópodos são semelhantes aos de Panopeus, e Eucratopsis Smith, 1869, que se parece com Panopeus tanto na forma dos pleópodos quanto em outros caracteres, sendo este o gênero mais bem característico do grupo.

A presença de Eucratopsis Smith, 1869, junto com os outros panopeíneos, faz com que, por prioridade, seja utilizada para esta subfamília o nome de Eucratopsinae.

O gênero Micropanope Stimpson, 1871, era anteriormente agrupado com os outros Eucratopsinae, porém estudos posteriores concluíram que eles se aproximam dos Trapeziinae (Pilumnidae). MARTIN (1984) analizando as zoeas de Xanthidae, separa Micropanope barbadensis (Rathbun, 1921) de outros xanthídeos. Contudo, em 1990, GUINOT analisa a posição sistemática dos Micropanope e transfere as espécies Micropanope spinipes (A. Milne Edwards, 1880), M. barbadensis e Micropanope fraseri (Garth, 1946) para o gênero Garthiope Guinot (1990); Micropanope xanthiformes (A. Milne Edwards, 1880) para Nanoplax Guinot (1967) e Micropanope distinctus (Rathbun, 1898) para Pseudomedaeus Guinot, 1968.

Neste mesmo trabalho, GUINOT resalta que Domecia Eudoux \& Souleyet, 1842, tem maiores afinidades com os Trapeziidae e não com os seus Menippidae. De acordo com os resultados obtidos, os Trapeziinae, representados então por Domecia, ficaram agrupados no ponto 12 da árvore, junto aos Pilumnus Leach, 1815. Com relação às afinidades propostas pela referida autora entre os Micropanope, Garthiope e trapeziíneos, e destes últimos aos Pilumnus, o nome Pilumnidae que tem prioridade sobre Trapeziidae, e a família deverá conter as subfamílias Pilumninae e Trapeziinae, inicialmente com os gêneros Pilumnus e Domecia, respectivamente. A posição dos outros gêneros dentro destas subfamílias ou até mesmo a criação de novas subfamílias, serão propostas posteriormente.

A inclusão dos Geryoninae entre os Goneplacidae foi, no passado, aceita por vários pesquisadores; ao contrário, sua classificação entre os Xanthidae foi sempre combatida. Do ponto de vista do desenvolvimento larval, RICE (1980) é da opinião de que estão mais próximos dos Portunidae que de qualquer outro grupo. Deixando os Portunidae de lado, a maior afinidade seria com os Goneplacidae. Estes mesmos, no entanto, estão pouco estudados quanto a este aspecto. 
Apesar do esforço realizado, não foi possível agrupar alguns dos gêneros da fauna brasileira classificados geralmente como Xanthidae ou Goneplacidae: Melybia Stimpson, 1871, Nanoplax Guinot, 1967, Cycloplax Guinot, 1969 e Tetraplax Rathbun, 1901.

Embora a classificação obtida concorde em suas grandes linhas com os resultados do estudo das larvas e com a percepção de numerosos pesquisadores anteriores, os estudos precisam continuar, seguindo agora também outras linhas, tais como: afinidades dos gêneros dentro de cada táxon; classificação dos outros Cancroidea com relação às famílias estudadas; classificação dos gêneros que não puderam ser classificados: descrição de novas espécies, etc.

\section{CONCLUSÕES}

A taxonomia numérica pode ser aplicada com proveito na classificação de crustáceos decápodos; - a análise fenética manteve a distinção da família Xanthidae tal como concebida tradicionalmente pelos autores (BALSS, 1955); - a análise cladística demonstrou que os Xanthidae são parafiléticos, devendo ser dividida em: Carpiliidae, Eriphiidae, Pilumnidae e Xanthidae s.s; - por motivos de prioridade a subfamília Panopeinae passa a se chamar Eucratopsinae; os Geryoninae são transferidos de Xanthidae para Goneplacidae; - a classificação obtida concorda em grande parte com o estudo das larvas.

\section{REFERÊNCIAS BIBLIOGRÁFICAS}

ABELE, L.G. \& W. KIN. 1986. An illustrated guide to the marine decapod crustaceans of Florida. State of Florida Departament of Environmental Regulation, Tallahassee (Techical Series), 8 (1).

ALCOCK, A. 1893. Materials for a carcinological fauna of India. 3. The Brachyura Cyclometopa. Part 1. The family Xanthidae. Jour. Asiatic Soc. Bengal, Calcutta, 67 (1): 67-233.

BALLS, H. 1957. Decapoda. VIII. Systematic. In: H.G. BRONH (ed.). Klassen und Ordnungen des Tierreichs 5, Abteilung 1, 7 (12): 1505-1672.

BENEDICT, J.E. \& M.J. RATHBUN. 1891. The genus Panopeus. Proc. U. S. Mus., Washington, 14 (858): 355-385.

BORRADAILE, L.A. 1907. On the classification of the decapod Crustaceans. Anns. \& Mag. Nat. Hist., London, (7) 19: 457-486.

CHACE JR., F.A.; J.J. MCDERMOTT; P.A. MCLAUGHLIN \& R.B. MANNING. 1986. Order Decapoda (Shrimps. Lobsters and Crabs). In: W. STERER (ed.). Marine Fauna And Flora Of Bermuda. New York, Wiley-Interscience.

DANA, J.D. 1852. Crustacea. Part 1. In: United States Exploring Expedition during the years 1838, 1839, 1840, 1841, 1842 under the Command of Charles Wilkes U. S. N., 13. Philadelphia.

FABRICIUS, J.C. 1781. Species insectorum exhibentes eorum differentias specificas, synonyma auctorum, loca natalia, metamorfhosis adjetis obser- 
vationibus, descriptionibus. Hamburgi.

FRANSOZO, A. 1987. Desenvolvimento larval de Eriphia gonagra (Fabricius, 1781) (Decapoda, Xanthidae), em laboratório. Revta Bras. Zool., São Paulo, 4 (3): 165-179.

GARTH, J.S. 1946. Littoral brachyuran fauna of the Galapagos Archipelago. Allan Hancock Pacific Expeditions 5 (10): 341-601.

GUINOT, D. 1966. Recherches preliminaires sur les groupements naturels chez les crustacés décapodes brachyures. I. Les affinités des genres Aethra, Osachila, Hepatus, Hepatella et Actaeomorpha. Bull. Mus. Nat. d'Hist. Nat., Paris, (2) 38 (5): 744-762.

.1967. Recherches preliminaires sur les groupements naturels chez les crustacés décapodes brachyures. I. Suite et fin. Bull. Mus. Nat. d'Hist. Nat., Paris, (2) 38 (6): 828-843.

-1968. Recherches preliminaires sur les groupements naturels chez les crustacés décapodes brachyures. IV. Observations sur quelques genres de Xanthidae. Bull. Mus. Nat. d'Hist. Nat., Paris, (2) 39 (4): 695-727 (1967).

1968. Recherches preliminaires sur les groupements naturels chez les crustacés décapodes brachyures. VI. Les Carpiliinae. Bull. Mus. Nat. d'Hist. Nat., Paris, (2) 40 (2): 320-334.

. 1969. Recherches preliminaires sur les groupements naturels chez les crustacés décapodes brachyures. VII. Les Goneplacidae (suite et fin). Bull. Mus. Nat. d'Hist. Nat., Paris, (2) 41: 688- 714.

. 1969. Sur divers Xanthidae, notamment sur Actaea de Haan et Paractaea Gen. Nov. (Crustacea Decapoda Brachyura). Cahiers du Pacifique 13: 223-267.

décapodes brachoures. I. La superfamille des Bellioidea et trois sousfamillies de Xanthoidea Xanthidae (Polydectinae Dana, Trichiinae de Haan, Actaeinae Alcock). Mém. Mus. Nat. d'Hist. Nat., Paris, n.s, 97 (A): 1- 308. décapodes brachoures. Comptes rendues hebdomadaires des Scéances de la Academie de Sciences, Paris, sér. D, 285: 1049-1052.

brachoures. Bull. Biol. France Belgique, Paris, n.sér, 112 (3): 211-293.

1990. Établissement du genre Garthiope gen. nov., ses Relations Avec le Genre Coralliope Guinot, 1967, et Leurs Affinités Avec les Trapeziidae sensu lato (Crustacea Decapoda Brachyura). Bull. Mus. Nat. Hist. Nat., Paris, (12) sec. A, 12 (2): 469-487.

DE HAAN, W. 1833-1850. Crustacea. In: P.F. VON SIEBOLD (ed.). Fauna japonica sive descriptio animalium, quae in itinere per Japonim, jussu et auspices superiorum, qui sumum in India Batava imperium tenent, suscepto, annis 1823-1830 collegit, notis, observationibus et adumbrationibus illustravit. Lugduni-Batavorum.

LATREILLE, P. A. 1817. Nouveau dictionaire d'histoire naturelle appliqué 
aux arts, à l'agriculture, à l'économie rural et domestique, à la medicine, etc...nouvelle édition presquàentirrement refondue et considérablement augmentée avec des figures tirées des trois régnes de la nature, 25: 610p.

MAC LEAY, W.S. 1838. On the brachyurous decapod Crustacea brought from the Cape by Dr. Smith, p.53-71. In: Illustrations of the Annulosa of South Africa... London.

MARTIN, J.W. 1984. Notes and bibliography on the larvae of xanthid crabs, with a key to the known xanthid zoeas of the western Atlantic and gulf of Mexico. Bull. Marine Sci., Miami, 34 (2): 220-239.

MARTIN, J.W. \& L.G. ABELE. 1986. Notes on male pleopod morphology in the brachyuran crab family Panopeid Ortmann, 1893, sensu Guinot (1978) (Decapoda). Crustaceana, Leiden, 50 (2): 182-198.

MIERS, E.J. 1886. Report on the Brachyura collected by H.M.S. Challenger during the years 1873-76. In: Reports on the Scientific Results of the Voyage of H.M.S. Challenger during the years 1873-76, Zoology, 17.

MILNE EDWARDS, A. 1893. Monographie des crustacés fossilis de la famille des Cancériens. Ann. Sc. nat., Zoologie, Paris, 20 (A): 273-384.

1869. Description d'un nouveau genre de crustacécancérien. Ann. Soc. Ent. France, Paris, 9 (4): 167-169.

MILNE EDWARDS, A. \& BOUVIER, E. L. 1900. Crustacés Décapodes. I. Brachyures et anomures. In: Expéditions scientifiques du Travailleur et du Talisman pendant les années 1880, 1881, 1882, 1883. Paris, Masson.

MILNE EDWARDS, H. 1834. Histoire naturelle des crustacés, comprenant l'anatomie, la physiologie et la classification de ces animaux. 1. Paris, Libraire Encyclopedie de Roret.

NG, P.K.L. \& G. RODRÍGUEZ. 1986. New records of Mimilambrus wileyi Williams, 1979 (Crustacea: Decapoda: Brachyura) with notes on the systematics of the Mimilambridae Williams, 1979, and Parthenopidae MacLeay, 1838, sensu Guinot, 1978. Proc. Biol. Soc. Wash. 99 (1): 88-99.

ORTMANN, A. 1893. Die Decapoden-Krebse des Strassburger Museums. VII. Abteilung Brachyura (Brachyura genuina Boas). II. Unterabteilung: Cancroidea, 2 Section: Cancrinea, 1. Gruppe: Cyclometopa. Zool. Jhrb., Berlin, 7: 411-495.

RATHBUN, M.J. 1897. A revision of the nomenclature of the Brachyura. Proc. Biol. Soc. Wash. 11 : 153-167.

- 1901. Results of the Branner-Agassiz Expedition to Brazil. I. The Decapoda and Stomatopoda Crustacea. Proc. Wash. Ac. Sc. 2: 133-156.

- 1901. The Brachyura and Macrura of Porto Rico. Bull. U.S. Fish Commission, Washington, 20 (2): 1-127, 129-137.

- 1914. New genera and species of american brachyrhynchous crabs. Proc. U.S. Nat. Mus., Washington, 47 (2047): 117-129.

- 1922. New species of crabs from Curacao. Proc. Biol. Soc. Wash. 35: 103-104.

1930. The cancroid crabs of America of the families Euryalidae, 
Portunidae, Atelecyclidae, Cancridae and Xanthidae. U.S. Nat. Mus. Bull., Washington, 152: 1-609.

RICE, A.L. 1980. Crab zoeal morphology and its bearing on thi classification of the Brachyura. Trans. Zool. Soc. London 35: 271-424.

SAMOUELLE, G. 1819. The entomologist's useful compendium, or an introduction to the knowledge of british insects. London.

SMITH, S.I. 1869. Notes on new little known species of American Cancroid Crustacea. Proc. Bost. Soc. Nat. Hist. 12: 274-289.

STIMPSON, W. 1857-1860. Prodromus descriptionis animalium evertebratorum, quae in expeditione ad Oceanum Pacificum Septentrionalem, a Republica Federata missa, Cadwaladaro Ringgold et Johanne Rodgers ducibus, observavit et descripsit. Proc. Ac. Nat. Sc. Philad. 9/12.

. 1871. Preliminary report on the Crustacea dredged in the Gulf Stream in the straits of Florida. Bull. Mus. Comp. Zool. Harvard Coll., Cambridge, 2: 109-160.

WILLIAMS, A.B. 1974. Allactaea lithostrota, a New Genus and Species of Crab (Decapoda: Xanthidae) from North Carolina, U.S.A. Proc. Biol. Soc. Wash. 87 (3): 16-26.

. 1984. Shrimps, lobsters and crabs of the atlantic coast of Eastern United States, Maine to Florida. Washington: Smithsonian Institution Press.

Recebido em 23.XI.1992; aceito em 21.II.1994. 